Mathematical Modelling and Analysis

Volume 21 Number 6, November 2016, 741-751

http://dx.doi.org/10.3846/13926292.2016.1237388

(c) Vilnius Gediminas Technical University, 2016
Publisher: Taylor\&Francis and VGTU

http://www.tandfonline.com/TMMA

ISSN: $1392-6292$

eISSN: 1648-3510

\title{
Dynamic Analysis for Bertrand Competition Model with Exponential Form*
}

\section{Huili $\mathrm{Ma}^{a}$ and Hui Feng ${ }^{a}$}

\author{
${ }^{a}$ College of Business, Northwest Normal University \\ 730070 Lanzhou Gansu, China \\ E-mail(corresp.): mahuili@nwnu.edu.cn
}

Received March 30, 2016; revised September 9, 2016; published online November 15, 2016

\begin{abstract}
This paper will consider a nonliear system of difference equations which describes a qualitative study of Bertrand oligopoly games with two boundedly rational players. With nonlinear demand function of exponential form, the local stability of equilibria and the global convergence of positive solutions for the dynamical system are analyzed.
\end{abstract}

Keywords: difference equations, boundedness, Bertrand model, global asymptotic behavior.

AMS Subject Classification: 39A11; 40A05; 91A25.

\section{Introduction}

Market economy is fundamentally a dynamic system, which can usually be described mathematically by difference equations. In the dynamic study of economics, a couple of economic models represented by difference equations are investigated, such as the classical cobweb model describing the variation of the supply and demand, the Cournot models of oligopoly, and so on [2], [1], [6], [11]. It is well known that Bertrand duopoly competition game is one of the basic oligopoly games with two players. In this paper we consider a Bertrand duopoly game with an industry where two firms produce heterogeneous products. Price strategic interactions appear because the total demand of the good depends on the price of the industry according to a given demand function. In the classical study of Bertrand game, the demand function is usually linear or quadratic. However the demand function of exponential form can describe the bounded rationality duopoly game more realistic [11] and at the same time the dynamic will be more complicated. We assume in this paper the demand function

$$
Q=f\left(p_{1}, p_{2}\right)=a \mathrm{e}^{-\left(p_{1}+p_{2}\right)},
$$

\footnotetext{
* This paper is funded by the National Natural Science Foundation of China(61363058), Natural Science Foundation of Gansu Province(145RJZA232) and Promotion Funds for Young Teachers in Northwest Normal University (NWNU-LKQN-12-14).
} 
where $a$ is a parameter of maximum demand in the market and $p_{i}, i=1,2$ denotes the price of the good produced by firm $i$. Assume the cost function

$$
C_{i}(Q)=c_{i} Q, \quad i=1,2,
$$

where $c_{i}$ is the marginal cost of the $i$ th firm. Then the profit resulting from the above Bertrand duopoly game is given by

$$
\Pi_{i}=p_{i} Q-c_{i} Q=a\left(p_{i}-c_{i}\right) \mathrm{e}^{-\left(p_{1}+p_{2}\right)} .
$$

The classical oligopoly games, and the associated notion of Nash equilibrium, are based on quite demanding notion of rationality. However, since the available information in the oligopoly market is incomplete, the rational players make their price decisions on a local estimate of the expected marginal profit $\frac{\partial \Pi_{i}}{\partial p_{i}}$. Hence, the dynamical equation of the bounded rationality player $i$ has the form

$$
p_{i}(t+1)=p_{i}(t)+\nu_{i} p_{i}(t) \frac{\partial \Pi_{i}}{\partial p_{i}}, \quad i=1,2,
$$

where $\nu_{i}, i=1,2$ is a positive parameter which represents the relative speed of the price adjustment by producer $i$.

Therefore, using (1.1)-(1.3), the discrete dynamical system becomes an iterated two-dimensional mapping which has the form

$$
\left\{\begin{array}{l}
p_{1}(t+1)=p_{1}(t)+a \nu_{1} \mathrm{e}^{-\left(p_{1}+p_{2}\right)} p_{1}(t)\left(1-p_{1}(t)\right)+a \nu_{1} c_{1} p_{1}(t) \mathrm{e}^{-\left(p_{1}+p_{2}\right)}, \\
p_{2}(t+1)=p_{2}(t)+a \nu_{2} \mathrm{e}^{-\left(p_{1}+p_{2}\right)} p_{2}(t)\left(1-p_{2}(t)\right)+a \nu_{1} c_{1} p_{2}(t) \mathrm{e}^{-\left(p_{1}+p_{2}\right)}
\end{array} .\right.
$$

We can rewrite this system in the new form

$$
\left\{\begin{array}{l}
x_{n+1}=x_{n}+\left(\alpha_{1}-\beta_{1} x_{n}\right) x_{n} \mathrm{e}^{-\left(x_{n}+y_{n}\right)} \\
y_{n+1}=y_{n}+\left(\alpha_{2}-\beta_{2} y_{n}\right) y_{n} \mathrm{e}^{-\left(x_{n}+y_{n}\right)}
\end{array}\right.
$$

where $x_{n}=p_{1}(t), y_{n}=p_{2}(t), \alpha_{i}=a \nu_{i}\left(1+c_{i}\right), \beta_{i}=a \nu_{i}, i=1,2$.

In this paper we study the boundedness and the global asymptotic behavior of the positive solutions of the system of difference equation

$$
\left\{\begin{array}{l}
x_{n+1}=x_{n}+\left(\alpha_{1}-\beta_{1} x_{n}\right) x_{n} \mathrm{e}^{-\left(x_{n}+y_{n}\right)} \\
y_{n+1}=y_{n}+\left(\alpha_{2}-\beta_{2} y_{n}\right) y_{n} \mathrm{e}^{-\left(x_{n}+y_{n}\right)}
\end{array}\right.
$$

where $\alpha_{i}, \beta_{i} \in(0, \infty)$ with $\alpha_{i}>\beta_{i}, i=1,2$, and the initial values $x_{0}, y_{0}$ are positive numbers.

In [5], by using the inverse demand function of exponential form, MF Elettreby and $\mathrm{H}$ El-Metwally studied a Cournot competition model described by difference systems of exponential form and they obtained the local stability of the equilibrium point and the global convergence of positive solutions. In [4], HA El-Metwally and AA Elsadany investigated the chaotic behavior of a duopoly Cournot game model of difference systems of exponential form. As for the studies of the behavior of positive solutions for difference equations of exponential form, we refer the readers to [3], [8], [9], [10] and the references therein. The next theorem will be a useful tool later in Section 3. 
Theorem 1. [7] Suppose $T=(f, g)$ be a monotone map on a closed and bounded rectangular region $S \subset \mathrm{R}^{2}$. If $T$ has a unique fixed point $E=(\bar{x}, \bar{y})$ in $S$, then $E$ is a global attractor of $T$ on $S$.

\section{Equilibria Points of (1.4)}

Observe that the equilibria points of system (1.4) are given by the equations

$$
\left\{\begin{array} { l } 
{ \overline { x } = \overline { x } + ( \alpha _ { 1 } - \beta _ { 1 } \overline { x } ) \overline { x } \mathrm { e } ^ { - ( \overline { x } + \overline { y } ) } , } \\
{ \overline { y } = \overline { y } + ( \alpha _ { 2 } - \beta _ { 2 } \overline { y } ) \overline { y } \mathrm { e } ^ { - ( \overline { x } + \overline { y } ) } , }
\end{array} \Rightarrow \left\{\begin{array}{l}
\left(\alpha_{1}-\beta_{1} \bar{x}\right) \bar{x} \mathrm{e}^{-(\bar{x}+\bar{y})}=0 \\
\left(\alpha_{2}-\beta_{2} \bar{y}\right) \bar{y} \mathrm{e}^{-(\bar{x}+\bar{y})}=0
\end{array}\right.\right.
$$

Obviously, system (1.4) has equilibria points $E_{0}=(0,0), E_{1}=\left(0, \frac{\alpha_{2}}{\beta_{2}}\right), E_{2}=$ $\left(\frac{\alpha_{1}}{\beta_{1}}, 0\right)$ and $E_{3}=\left(\frac{\alpha_{1}}{\beta_{1}}, \frac{\alpha_{2}}{\beta_{2}}\right)$.

In the following, we deal with the local stability of the above four equilibrium points of (1.4). Now set $F\left(x_{n}, y_{n}\right)=x_{n}+\left(\alpha_{1}-\beta_{1} x_{n}\right) x_{n} \mathrm{e}^{-\left(x_{n}+y_{n}\right)}$, $G\left(x_{n}, y_{n}\right)=y_{n}+\left(\alpha_{2}-\beta_{2} y_{n}\right) y_{n} \mathrm{e}^{-\left(x_{n}+y_{n}\right)}$, where $F(x, y)$ and $G(x, y)$ are continuous functions. Then we obtain

$$
\begin{aligned}
& \frac{\partial F(x, y)}{\partial x}=1+\left[\left(\alpha_{1}-\beta_{1} x\right)-\beta_{1} x-\left(\alpha_{1}-\beta_{1} x\right) x\right] \mathrm{e}^{-(x+y)}, \\
& \frac{\partial F(x, y)}{\partial y}=-\left(\alpha_{1}-\beta_{1} x\right) x \mathrm{e}^{-(x+y)}, \quad \frac{\partial G(x, y)}{\partial x}=-\left(\alpha_{2}-\beta_{2} y\right) y \mathrm{e}^{-(x+y)}, \\
& \frac{\partial G(x, y)}{\partial y}=1+\left[\left(\alpha_{2}-\beta_{2} y\right)-\beta_{2} y-\left(\alpha_{2}-\beta_{2} y\right) y\right] \mathrm{e}^{-(x+y)} .
\end{aligned}
$$

Proposition 1. The equilibrium point $E_{0}$ of system (1.4) is unstable.

Proof. We compute the Jacobian matrix of system (1.4) about the equilibrium point $E_{0}=(0,0)$ and have

$$
J\left(E_{0}\right)=\left(\begin{array}{cc}
1+\alpha_{1} & 0 \\
0 & 1+\alpha_{2}
\end{array}\right) .
$$

Therefore the eigenvalues of $J\left(E_{0}\right)$ are given by $\lambda_{1}=1+\alpha_{1}$ and $\lambda_{2}=1+\alpha_{2}$. Then $\left|\lambda_{i}\right|>1, i=1,2$. So the equilibrium point $E_{0}$ of system (1.4) is unstable. The proof is completed.

Proposition 2. The equilibria points $E_{1}$ and $E_{2}$ of system (1.4) are saddle points.

Proof. The Jacobian matrix of system (1.4) about the equilibrium point $E_{1}=$ $\left(0, \alpha_{2} / \beta_{2}\right)$ has the form

$$
J\left(E_{1}\right)=\left(\begin{array}{cc}
1+\alpha_{1} \mathrm{e}^{-\alpha_{2} / \beta_{2}} & 0 \\
0 & 1-\alpha_{2} \mathrm{e}^{-\alpha_{2} / \beta_{2}}
\end{array}\right) .
$$

Therefore the eigenvalues of $J\left(E_{1}\right)$ are given by $\lambda_{1}=1+\alpha_{1} \mathrm{e}^{-\alpha_{2} / \beta_{2}}$ and $\lambda_{2}=$ $1-\alpha_{2} \mathrm{e}^{-\alpha_{2} / \beta_{2}}$. Then

$$
\left|\lambda_{1}\right|>1, \quad\left|\lambda_{2}\right|<1
$$


Thus it follows that the equilibrium point $E_{1}$ of system (1.1) is a saddle point. Similarly, one can easily prove that the equilibrium point $E_{2}$ of system (1.4) is also a saddle point. This completes the proof.

Proposition 3. The Nash equilibrium point $E_{3}$ of system (1.4) is asymptotically stable if either $0<\alpha_{i} \mathrm{e}^{-\left(\frac{\alpha_{1}}{\beta_{1}}+\frac{\alpha_{2}}{\beta_{2}}\right)}<1$ or $1<\alpha_{i} \mathrm{e}^{-\left(\frac{\alpha_{1}}{\beta_{1}}+\frac{\alpha_{2}}{\beta_{2}}\right)}<2, i=1,2$.

Proof. The Jacobian matrix of system (1.4) about the equilibrium point $E_{3}=$ $\left(\alpha_{1} / \beta_{1}, \alpha_{2} / \beta_{2}\right)$ is

$$
J\left(E_{1}\right)=\left(\begin{array}{cc}
1-\alpha_{1} \mathrm{e}^{-\left(\frac{\alpha_{1}}{\beta_{1}}+\frac{\alpha_{2}}{\beta_{2}}\right)} & 0 \\
0 & 1-\alpha_{2} \mathrm{e}^{-\left(\frac{\alpha_{1}}{\beta_{1}}+\frac{\alpha_{2}}{\beta_{2}}\right)}
\end{array}\right) .
$$

Therefore the eigenvalues of $J\left(E_{3}\right)$ are given by

$$
\lambda_{1}=1-\alpha_{1} \mathrm{e}^{-\left(\frac{\alpha_{1}}{\beta_{1}}+\frac{\alpha_{2}}{\beta_{2}}\right)}, \quad \lambda_{2}=1-\alpha_{2} \mathrm{e}^{-\left(\frac{\alpha_{1}}{\beta_{1}}+\frac{\alpha_{2}}{\beta_{2}}\right)} .
$$

It is well known that the equilibrium point $E_{3}$ of system (1.4) is locally asymptotically stable if for $i=1,2,\left|\lambda_{i}\right|<1$ are satisfied, which is true when $0<\alpha_{i} \mathrm{e}^{-\left(\frac{\alpha_{1}}{\beta_{1}}+\frac{\alpha_{2}}{\beta_{2}}\right)}<1$ or $1<\alpha_{i} \mathrm{e}^{-\left(\frac{\alpha_{1}}{\beta_{1}}+\frac{\alpha_{2}}{\beta_{2}}\right)}<2, i=1,2$. The proof is completed.

\section{Global Stability Analysis of (1.4)}

In this section we first concern with the boundedness properties of the positive solutions for system (1.4). Under appropriate conditions, we give some bounded results related to system (1.4).

Theorem 2. Assume that $\beta_{i} \mathrm{e}^{-\frac{\alpha_{i}+\beta_{i}}{\beta_{i}}}<1$. Then every solution $\left\{\left(x_{n}, y_{n}\right)\right\}_{n=0}^{\infty}$ of system (1.4) with $x_{0}>0$ and $y_{0}>0$, satisfies that $x_{n}>0$ and $y_{n}>0$ for all $n>0$.

Proof. Let $H_{i}(x, y), i=1,2$ be continuous functions defined by

$$
H_{i}(x, y)=1+\left(\alpha_{i}-\beta_{i} x\right) \mathrm{e}^{-(x+y)}, i=1,2 .
$$

Then system (1.4) can be rewritten in the form

$$
x_{n+1}=x_{n} H_{1}\left(x_{n}, y_{n}\right), \quad y_{n+1}=y_{n} H_{2}\left(x_{n}, y_{n}\right) .
$$

Now assume that $\left\{\left(x_{n}, y_{n}\right)\right\}_{n=0}^{\infty}$ is a solution of system (1.4) with positive initial values. Then it suffices to show that $H_{i}(x, y), i=1,2$ are positive for all $x_{0}>0$ and $y_{0}>0$. Observe that

$$
\frac{\partial H_{i}(x, y)}{\partial x}=\left(-\beta_{i}-\alpha_{i}+\beta_{i} x\right) \mathrm{e}^{-(x+y)}, \frac{\partial H_{i}(x, y)}{\partial y}=-\left(\alpha_{i}-\beta_{i} x\right) \mathrm{e}^{-(x+y)}, i=1,2 .
$$

Therefore $H_{i}$ have no positive critical points. Let $a$ and $b$ be arbitrary positive numbers and consider the domain

$$
D=\{(x, y): 0 \leq x \leq a, 0 \leq y \leq b\} .
$$


Then for $i=1,2$, we see that

$$
\begin{aligned}
& H_{i}(0, y)=1+\alpha_{i} \mathrm{e}^{-y}, \quad H_{i}(a, y)=1+\left(\alpha_{i}-a \beta_{i}\right) \mathrm{e}^{-(a+y)}, \quad 0 \leq y \leq b, \\
& H_{i}(x, 0)=1+\left(\alpha_{i}-\beta_{i} x\right) \mathrm{e}^{-x}, H_{i}(x, b)=1+\left(\alpha_{i}-\beta_{i} x\right) \mathrm{e}^{-(x+b)}, 0 \leq x \leq a .
\end{aligned}
$$

Using elementary differential calculus, we obtain that the absolute minimum of $H_{i}$ is $1-\beta_{i} \mathrm{e}^{-\frac{\alpha_{i}+\beta_{i}}{\beta_{i}}}$. Therefore $H_{i}(x, y) \geq 1-\beta_{i} \mathrm{e}^{-\frac{\alpha_{i}+\beta_{i}}{\beta_{i}}}>0$ for all $(x, y) \in D$. Since $a$ and $b$ are arbitrary positive numbers, we can conclude that $H_{i}(x, y)>0$ for $i=1,2$ and for all $(x, y) \in(0, \infty)^{2}$.

Theorem 3. Let $\left\{\left(x_{n}, y_{n}\right)\right\}_{n=0}^{\infty}$ be a solution of system (1.4) with $\left(x_{n_{0}}, y_{n_{0}}\right) \in$ $\left(0, \frac{\alpha_{1}}{\beta_{1}}\right] \times\left(0, \frac{\alpha_{2}}{\beta_{2}}\right]$ for some $n_{0} \geq 0$. If for $i=1,2, \alpha_{i}<1$, then $\left\{\left(x_{n}, y_{n}\right)\right\} \in$ $\left(0, \frac{\alpha_{1}}{\beta_{1}}\right] \times\left(0, \frac{\alpha_{2}}{\beta_{2}}\right]$ for all $n \geq n_{0}$.

Proof. Let $n_{0} \geq 0$ be such that $x_{n_{0}} \in\left(0, \frac{\alpha_{1}}{\beta_{1}}\right]$. It follows from system (1.4) that

$$
x_{n_{0}+1} \leq x_{n_{0}}+\left(\alpha_{1}-\beta_{1} x_{n_{0}}\right) x_{n_{0}} .
$$

Set $h(x)=x+\left(\alpha_{1}-\beta_{1} x\right) x$. We obtain that

$$
h^{\prime}(x)=1+\alpha_{1}-2 \beta_{1} x, \quad h^{\prime \prime}(x)=-2 \beta_{1}<0 .
$$

Hence $h^{\prime}(x)$ is decreasing on $\left(0, \frac{\alpha_{1}}{\beta_{1}}\right]$. Then $h^{\prime}\left(\frac{\alpha_{1}}{\beta_{1}}\right)=1-\alpha_{1}>0$. Therefore $h(x)$ is increasing on $\left(0, \frac{\alpha_{1}}{\beta_{1}}\right]$. Consequently, $h(x) \leq h\left(\frac{\alpha_{1}}{\beta_{1}}\right)=\frac{\alpha_{1}}{\beta_{1}}$. So $x_{n} \leq \frac{\alpha_{1}}{\beta_{1}}$ for all $n \geq n_{0}$. Similar method can be applied for $y_{n}$. This completes the proof.

Theorem 4. Assume that $\alpha_{i}<1$. Then every solution $\left\{\left(x_{n}, y_{n}\right)\right\}_{n=0}^{\infty}$ of system (1.4) satisfies

$$
\begin{aligned}
& x_{n} \leq\left(1-\alpha_{1}\right)^{n-n_{0}} x_{n_{0}}+\frac{\alpha_{1}}{\beta_{1}}\left[1-\left(1-\alpha_{1}\right)^{n-n_{0}}\right], \\
& y_{n} \leq\left(1-\alpha_{1}\right)^{n-n_{0}} y_{n_{0}}+\frac{\alpha_{1}}{\beta_{1}}\left[1-\left(1-\alpha_{1}\right)^{n-n_{0}}\right]
\end{aligned}
$$

for any $n_{0}$ satisfying $x_{n_{0}} \in\left(0, \frac{\alpha_{1}}{\beta_{1}}\right], y_{n_{0}} \in\left(0, \frac{\alpha_{2}}{\beta_{2}}\right]$.

Proof. Let $n \geq 0$ be such that $x_{n} \in\left(0, \frac{\alpha_{1}}{\beta_{1}}\right], y_{n} \in\left(0, \frac{\alpha_{2}}{\beta_{2}}\right]$. It follows from system (1.4) that

$$
x_{n+1} \leq x_{n}+\left(\alpha_{1}-\beta_{1} x_{n}\right) x_{n} \leq x_{n}+\left(\alpha_{1}-\beta_{1} x_{n}\right) \frac{\alpha_{1}}{\beta_{1}}=\left(1-\alpha_{1}\right) x_{n}+\frac{\alpha_{1}^{2}}{\beta_{1}} .
$$

Since $\alpha_{1}<1$, we have

$$
x_{n+2} \leq\left(1-\alpha_{1}\right) x_{n+1}+\frac{\alpha_{1}^{2}}{\beta_{1}}=\left(1-\alpha_{1}\right)^{2} x_{n}+\left(1-\alpha_{1}\right) \frac{\alpha_{1}^{2}}{\beta_{1}}+\frac{\alpha_{1}^{2}}{\beta_{1}} .
$$


By induction, for fixed $n_{0}$ satisfying $x_{n_{0}} \in\left(0, \alpha_{1} / \beta_{1}\right], y_{n_{0}} \in\left(0, \alpha_{2} / \beta_{2}\right]$, we obtain

$$
\begin{aligned}
x_{n} & \leq\left(1-\alpha_{1}\right)^{n-n_{0}} x_{n_{0}}+\frac{\alpha_{1}^{2}}{\beta_{1}}\left[\left(1-\alpha_{1}\right)^{n-n_{0}-1}+\left(1-\alpha_{1}\right)^{n-n_{0}-2}+\cdots+\left(1-\alpha_{1}\right)+1\right] \\
& =\left(1-\alpha_{1}\right)^{n-n_{0}} x_{n_{0}}+\frac{\alpha_{1}^{2}}{\beta_{1}} \frac{1-\left(1-\alpha_{1}\right)^{n-n_{0}}}{\alpha_{1}} \\
& =\left(1-\alpha_{1}\right)^{n-n_{0}} x_{n_{0}}+\frac{\alpha_{1}}{\beta_{1}}\left[1-\left(1-\alpha_{1}\right)^{n-n_{0}}\right] .
\end{aligned}
$$

Similarly, we could prove

$$
y_{n} \leq\left(1-\alpha_{1}\right)^{n-n_{0}} y_{n_{0}}+\frac{\alpha_{1}}{\beta_{1}}\left[1-\left(1-\alpha_{1}\right)^{n-n_{0}}\right]
$$

for any $n_{0}$ satisfying $x_{n_{0}} \in\left(0, \frac{\alpha_{1}}{\beta_{1}}\right], y_{n_{0}} \in\left(0, \frac{\alpha_{2}}{\beta_{2}}\right]$.

The following corollary is coming immediately from Theorem 4.

Corollary 1. Assume $\alpha_{i}<1, i=1,2$. Then for any initial point $\left(x_{n_{0}}, y_{n_{0}}\right) \in$ $\left(0, \alpha_{1} / \beta_{1}\right] \times\left(0, \alpha_{2} / \beta_{2}\right]$, every positive solution $\left\{\left(x_{n}, y_{n}\right)\right\}_{n=0}^{\infty}$ of system $(1.4)$ is bounded. Moreover,

$$
\limsup _{n \rightarrow \infty} x_{n} \leq \alpha_{1} / \beta_{1}, \quad \limsup _{n \rightarrow \infty} y_{n} \leq \alpha_{2} / \beta_{2} .
$$

Next, in the following theorem, we investigate the global attractivity of the equilibrium point $(\bar{x}, \bar{y})$ of system (1.4).

Theorem 5. Assume that $\alpha_{i}<1$. Then the unique positive equilibrium point $(\bar{x}, \bar{y})$ of system (1.4) is a global attractor of all positive solutions of system $(1.4)$.

Proof. Let $\left\{\left(x_{n}, y_{n}\right)\right\}_{n=0}^{\infty}$ be a solution of system (1.4). In the first case, if $x_{0} \leq \bar{x}<\frac{\alpha_{1}}{\beta_{1}}$,

$$
x_{1}=x_{0}+\left(\alpha_{1}-\beta_{1} x_{0}\right) x_{0} \mathrm{e}^{-\left(x_{0}+y_{0}\right)}>x_{0} .
$$

Then the sequence $\left\{x_{n}\right\}_{n=0}^{\infty}$ is increasing and since it was shown that it is bounded above by $\frac{\alpha_{1}}{\beta_{1}}$, then it converges to the unique positive equilibrium point $\bar{x}$. The second case is $x_{0} \geq \bar{x}$, we will show that there exists a positive integer $N$ such that $x_{N} \leq \bar{x}$. Notice that in this case

$$
x_{1}=x_{0}+\left(\alpha_{1}-\beta_{1} x_{0}\right) x_{0} \mathrm{e}^{-\left(x_{0}+y_{0}\right)}<x_{0} .
$$

Repeating this step confirms that $\left\{x_{n}\right\}$ is a decreasing sequence. Thus either there exists $N \in \mathbb{N}$ such that $x_{N}<\bar{x}$ or $x_{n}$ is a bounded decreasing sequence which has a limit $d_{1}>\bar{x}$. In whichever cases, we can assume similarly that $y_{n}$ has a limit $d_{2}$. Set $f(x, y)=\left(\beta_{1} x-\alpha_{1}\right) x \mathrm{e}^{-(x+y)}$. By the continuity of $f$ and convergence of $\left(x_{n}, y_{n}\right)$, we have there exists $n_{1} \in \mathbb{N}$ such that for any $n \geq n_{1}$, $f\left(x_{n}, y_{n}\right) \geq \delta$, where $\delta=\frac{f\left(d_{1}, d_{2}\right)}{2}>0$. Therefore, for any $n \geq n_{1}$, $x_{n+1}=x_{n}+\left(\alpha_{1}-\beta_{1} x_{n}\right) x_{n} \mathrm{e}^{-\left(x_{n}+y_{n}\right)}=x_{n}-\left(\beta_{1} x_{n}-\alpha_{1}\right) x_{n} \mathrm{e}^{-\left(x_{n}+y_{n}\right)} x_{n}<x_{n}-\delta$. 
So there exists a positive integer $N$ such that $x_{N}<\bar{x}$.

Similarly, it can be shown that the sequence $\left\{y_{n}\right\}_{n=0}^{\infty}$ converges to the unique positive equilibrium point $\bar{y}$. Thus $\left\{\left(x_{n}, y_{n}\right)\right\}_{n=0}^{\infty}$ converges to $(\bar{x}, \bar{y})$.

Example 1. Set $\alpha_{1}=0.6, \alpha_{2}=0.5,, \beta_{1}=0.3, \beta_{2}=0.1$, Figure 1 shows the stability of equilibrium of (1.4).

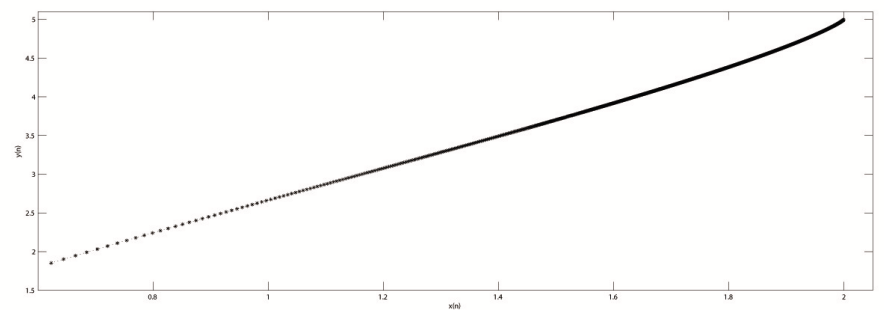

Figure 1. The stability of equilibrium of (1.4) while $\alpha_{i}>\beta_{i}$

As a special case at last, when $\alpha_{1}=\alpha_{2}=\alpha, \beta_{1}=\beta_{2}=\beta$, we contemplate the following system

$$
\left\{\begin{array}{l}
x_{n+1}=x_{n}+\left(\alpha-\beta x_{n}\right) x_{n} \mathrm{e}^{-\left(x_{n}+y_{n}\right)}, \\
y_{n+1}=y_{n}+\left(\alpha-\beta y_{n}\right) y_{n} \mathrm{e}^{-\left(x_{n}+y_{n}\right)}
\end{array}\right.
$$

we can have

Theorem 6. Assume that $\alpha<1$. If $\beta>\frac{\alpha^{2}+4 \beta^{2}}{4}$, then the unique positive equilibrium point $(\bar{x}, \bar{y})$ of system (1.4) is a global attractor of all positive solutions of system (3.1).

Proof. Rewrite (3.1) as

$$
x_{n+1}=F\left(x_{n}, y_{n}\right), \quad y_{n+1}=G\left(x_{n}, y_{n}\right),
$$

where $F(x, y)=x+(\alpha-\beta x) x \mathrm{e}^{-(x+y)}$ and $G(x, y)=y+(\alpha-\beta y) y \mathrm{e}^{-(x+y)}$ are continuous functions. Now consider

$$
\begin{cases}m_{1}=F\left(m_{1}, M_{2}\right), & M_{1}=F\left(M_{1}, m_{2}\right), \\ m_{2}=G\left(M_{1}, m_{2}\right), & M_{2}=G\left(m_{1}, M_{2}\right) .\end{cases}
$$

That is,

$$
\begin{cases}m_{1}=m_{1}+\left(\alpha-\beta m_{1}\right) m_{1} \mathrm{e}^{-\left(m_{1}+M_{2}\right)}, & M_{1}=M_{1}+\left(\alpha-\beta M_{1}\right) M_{1} \mathrm{e}^{-\left(M_{1}+m_{2}\right)}, \\ m_{2}=m_{2}+\left(\alpha-\beta m_{2}\right) m_{2} \mathrm{e}^{-\left(M_{1}+m_{2}\right)}, & M_{2}=M_{2}+\left(\alpha-\beta M_{2}\right) M_{2} \mathrm{e}^{-\left(m_{1}+M_{2}\right)} .\end{cases}
$$

Obviously, $m_{1}=M_{1}=m_{2}=M_{2}$ satisfy all the above equations. Solving these equations directly can obtain $m_{1}=M_{1}=m_{2}=M_{2}=\alpha / \beta$. So (3.1) has a unique fixed point $(\bar{x}, \bar{y})=(\alpha / \beta, \alpha / \beta)$. 
On the other hand, we can see

$$
\frac{\partial F}{\partial x}=1+\left[\alpha-(\alpha+2 \beta) x+\beta x^{2}\right] \mathrm{e}^{-(x+y)} \geq 1-\frac{\alpha^{2}+4 \beta^{2}}{4 \beta} .
$$

Therefore, $\frac{\partial F}{\partial x}>0$ if $\beta>\frac{\alpha^{2}+4 \beta^{2}}{4}$. We can do the same way to get that $\frac{\partial G}{\partial x}>0$. Thus, by Theorem 1, it follows that the unique positive equilibrium point $(\bar{x}, \bar{y})$ is a global attractor of all positive solutions of system (3.1).

\section{Remark}

In this last section, just from the mathematics point of view, we give a remark to discuss the case $\alpha_{i} \leq \beta_{i}, i=1,2$. We will see the unique positive equilibrium point $(\bar{x}, \bar{y})$ of system $(1.4)$ is a global attractor of all positive solutions of system (1.4).

Proposition 4. Let $\left\{\left(x_{n}, y_{n}\right)\right\}_{n=0}^{\infty}$ be a solution of system (1.4) with $\left(x_{n_{0}}, y_{n_{0}}\right)$ $\in\left(0, \frac{\alpha_{1}}{\beta_{1}}\right] \times\left(0, \frac{\alpha_{2}}{\beta_{2}}\right]$ for some $n_{0} \geq 0$. If for $i=1,2$, one of the followings is hold: (i) $\alpha_{i} \leq \beta_{i}, \quad \beta_{i} \mathrm{e}^{-\frac{\alpha_{i}}{\beta_{i}}}<1 ; \quad$ (ii) $\alpha_{i}<1$, then $\left\{\left(x_{n}, y_{n}\right)\right\} \in\left(0, \frac{\alpha_{1}}{\beta_{1}}\right] \times\left(0, \frac{\alpha_{2}}{\beta_{2}}\right]$ for all $n \geq n_{0}$.

Proof. For condition (i), we can prove as follows. Let $n_{0} \geq 0$ be such that $x_{n_{0}} \in\left(0, \frac{\alpha_{1}}{\beta_{1}}\right]$. Since $\alpha_{i} \leq \beta_{i}$ in (i), it follows from system (1.4) that

$$
x_{n_{0}+1} \leq x_{n_{0}}+\left(\alpha_{1}-\beta_{1} x_{n_{0}}\right) \mathrm{e}^{-\frac{\alpha_{1}}{\beta_{1}}} .
$$

Set $g(x)=x+\left(\alpha_{1}-\beta_{1} x\right) \mathrm{e}^{-\alpha_{1} / \beta_{1}}$. Then $g^{\prime}(x)=1-\beta_{1} \mathrm{e}^{-\frac{\alpha_{1}}{\beta_{1}}}$. If (i) is hold, we have $g^{\prime}(x)>0$, and then $g(x)$ is increasing on $\left(0, \frac{\alpha_{1}}{\beta_{1}}\right]$. Consequently, $g(x) \leq$ $g\left(\frac{\alpha_{1}}{\beta_{1}}\right)=\frac{\alpha_{1}}{\beta_{1}}$. Similar method can be applied for $y_{n}$.

As for condition (ii), we have the following proof. Let $n_{0} \geq 0$ be such that $x_{n_{0}} \in\left(0, \frac{\alpha_{1}}{\beta_{1}}\right]$. It follows from system (1.4) that

$$
x_{n_{0}+1} \leq x_{n_{0}}+\left(\alpha_{1}-\beta_{1} x_{n_{0}}\right) x_{n_{0}} .
$$

Set $h(x)=x+\left(\alpha_{1}-\beta_{1} x\right) x$. Then

$$
h^{\prime}(x)=1+\alpha_{1}-2 \beta_{1} x, \quad h^{\prime \prime}(x)=-2 \beta_{1}<0 .
$$

So $h^{\prime}(x)$ is decreasing on $\left(0, \frac{\alpha_{1}}{\beta_{1}}\right]$. While $h^{\prime}\left(\frac{\alpha_{1}}{\beta_{1}}\right)=1-\alpha_{1}>0$ since (ii) is hold. Therefore, $h^{\prime}(x)>0$ on $\left(0, \frac{\alpha_{1}}{\beta_{1}}\right]$, that is, $h(x)$ is increasing on $\left(0, \frac{\alpha_{1}}{\beta_{1}}\right]$. Hence $h(x) \leq h\left(\alpha_{1} / \beta_{1}\right)=\alpha_{1} / \beta_{1}$. Similar method can be applied for $y_{n}$.

Proposition 5. Assume for $i=1,2, \alpha_{i} \mathrm{e}^{-\frac{\alpha_{i}}{\beta_{i}}}<1$. Then every solution $\left\{\left(x_{n}, y_{n}\right)\right\}_{n=0}^{\infty}$ of system (1.4) satisfies

$$
\begin{aligned}
& x_{n} \leq\left(1-\alpha_{1} \mathrm{e}^{-\frac{\alpha_{1}}{\beta_{1}}}\right)^{n-n_{0}} x_{n_{0}}+\frac{\alpha_{1}}{\beta_{1}}\left[1-\left(1-\alpha_{1} \mathrm{e}^{-\frac{\alpha_{1}}{\beta_{1}}}\right)^{n-n_{0}}\right], \\
& y_{n} \leq\left(1-\alpha_{2} \mathrm{e}^{-\frac{\alpha_{2}}{\beta_{2}}}\right)^{n-n_{0}} y_{n_{0}}+\frac{\alpha_{2}}{\beta_{2}}\left[1-\left(1-\alpha_{2} \mathrm{e}^{-\frac{\alpha_{2}}{\beta_{2}}}\right)^{n-n_{0}}\right],
\end{aligned}
$$

for any $n_{0}$ satisfying $x_{n_{0}} \in\left(0, \alpha_{1} / \beta_{1}\right], y_{n_{0}} \in\left(0, \alpha_{2} / \beta_{2}\right]$. 
Proof. Let $n \geq 0$ be such that $x_{n} \in\left(0, \frac{\alpha_{1}}{\beta_{1}}\right]$. It follows from system (1.4) that

$$
x_{n+1} \leq x_{n}+\left(\alpha_{1}-\beta_{1} x_{n}\right) x_{n} \mathrm{e}^{-x_{n}} .
$$

Since $\alpha_{1} / \beta_{1} \leq 1$, we have

$$
x_{n+1} \leq x_{n}+\left(\alpha_{1}-\beta_{1} x_{n}\right) \frac{\alpha_{1}}{\beta_{1}} \mathrm{e}^{-\frac{\alpha_{1}}{\beta_{1}}}=\left(1-\alpha_{1} \mathrm{e}^{-\frac{\alpha_{1}}{\beta_{1}}}\right) x_{n}+\frac{\alpha_{1}^{2}}{\beta_{1}} \mathrm{e}^{-\frac{\alpha_{1}}{\beta_{1}}} .
$$

And if $\alpha_{i} \mathrm{e}^{-\alpha_{i} / \beta_{i}}<1$, then

$$
x_{n+2} \leq\left(1-\alpha_{1} \mathrm{e}^{-\frac{\alpha_{1}}{\beta_{1}}}\right)^{2} x_{n}+\frac{\alpha_{1}^{2}}{\beta_{1}} \mathrm{e}^{-\frac{\alpha_{1}}{\beta_{1}}}\left(1-\alpha_{1} \mathrm{e}^{-\frac{\alpha_{1}}{\beta_{1}}}\right)+\frac{\alpha_{1}^{2}}{\beta_{1}} \mathrm{e}^{-\frac{\alpha_{1}}{\beta_{1}}} .
$$

By induction, for fixed $n_{0}$ satisfying $x_{n_{0}} \in\left(0, \alpha_{1} / \beta_{1}\right]$, we obtain

$$
\begin{aligned}
x_{n} \leq & \left(1-\alpha_{1} \mathrm{e}^{-\frac{\alpha_{1}}{\beta_{1}}}\right)^{n-n_{0}} x_{n_{0}}+\frac{\alpha_{1}^{2}}{\beta_{1}} \mathrm{e}^{-\frac{\alpha_{1}}{\beta_{1}}}\left[\left(1-\alpha_{1} \mathrm{e}^{-\frac{\alpha_{1}}{\beta_{1}}}\right)^{n-n_{0}-1}\right. \\
& \left.+\left(1-\alpha_{1} \mathrm{e}^{-\frac{\alpha_{1}}{\beta_{1}}}\right)^{n-n_{0}-2}+\cdots+\left(1-\alpha_{1} \mathrm{e}^{-\frac{\alpha_{1}}{\beta_{1}}}\right)+1\right] \\
= & \left(1-\alpha_{1} \mathrm{e}^{-\frac{\alpha_{1}}{\beta_{1}}}\right)^{n-n_{0}} x_{n_{0}}+\frac{\alpha_{1}^{2}}{\beta_{1}} \mathrm{e}^{-\frac{\alpha_{1}}{\beta_{1}}} \frac{1-\left(1-\alpha_{1} \mathrm{e}^{-\frac{\alpha_{1}}{\beta_{1}}}\right)^{n-n_{0}}}{\alpha_{1} \mathrm{e}^{-\frac{\alpha_{1}}{\beta_{1}}}} \\
= & \left(1-\alpha_{1} \mathrm{e}^{-\frac{\alpha_{1}}{\beta_{1}}}\right)^{n-n_{0}} x_{n_{0}}+\frac{\alpha_{1}}{\beta_{1}}\left[1-\left(1-\alpha_{1} \mathrm{e}^{-\frac{\alpha_{1}}{\beta_{1}}}\right)^{n-n_{0}}\right] .
\end{aligned}
$$

Similarly, we could prove

$$
y_{n} \leq\left(1-\alpha_{2} \mathrm{e}^{-\frac{\alpha_{2}}{\beta_{2}}}\right)^{n-n_{0}} y_{n_{0}}+\frac{\alpha_{2}}{\beta_{2}}\left[1-\left(1-\alpha_{2} \mathrm{e}^{-\frac{\alpha_{2}}{\beta_{2}}}\right)^{n-n_{0}}\right]
$$

for any $n_{0}$ satisfying $x_{n_{0}} \in\left(0, \alpha_{1} / \beta_{1}\right], y_{n_{0}} \in\left(0, \alpha_{2} / \beta_{2}\right]$.

The following corollary is coming immediately from Proposition 5.

Corollary 2. Assume $\alpha_{i} \mathrm{e}^{-\frac{\alpha_{i}}{\beta_{i}}}<1, i=1,2$. Then for any initial point $\left(x_{n_{0}}, y_{n_{0}}\right)$ $\in\left(0, \alpha_{1} / \beta_{1}\right] \times\left(0, \alpha_{2} / \beta_{2}\right]$, every positive solution $\left\{\left(x_{n}, y_{n}\right)\right\}_{n=0}^{\infty}$ of system (1.4) is bounded. Moreover,

$$
\limsup _{n \rightarrow \infty} x_{n} \leq \alpha_{1} / \beta_{1}, \quad \limsup _{n \rightarrow \infty} y_{n} \leq \alpha_{2} / \beta_{2} .
$$

Next, in the following theorem, we investigate the global attractivity of the equilibrium point $(\bar{x}, \bar{y})$ of system (1.4).

Theorem 7. Assume that $\alpha_{i} \leq \beta_{i}, \alpha_{i} \mathrm{e}^{-\frac{\alpha_{i}}{\beta_{i}}}<1$. Then the unique positive equilibrium point $(\bar{x}, \bar{y})$ of system (1.4) is a global attractor of all positive solutions of system (3.1).

Proof. Let $\left\{\left(x_{n}, y_{n}\right)\right\}_{n=0}^{\infty}$ be a solution of system (1.4) and let $x_{n} \leq \bar{x}<\frac{\alpha_{1}}{\beta_{1}}$ (the case whenever $x_{n} \geq \bar{x}$ is similar and so it will be omitted). Thus

$$
\frac{\alpha_{1}}{\beta_{1}} \geq x_{n+1}=x_{n}+\left(\alpha_{1}-\beta_{1} x_{n}\right) x_{n} \mathrm{e}^{-\left(x_{n}+y_{n}\right)} \geq x_{n} .
$$


Then the sequence $\left\{x_{n}\right\}_{n=0}^{\infty}$ is increasing and since it was shown that it is bounded above, then it converges to the unique positive equilibrium point $\bar{x}$. Similarly, it is easy to show that the sequence $\left\{y_{n}\right\}_{n=0}^{\infty}$ is increasing and since it was shown that it is bounded above, then it converges to the unique positive equilibrium point $\bar{y}$. Thus $\left\{\left(x_{n}, y_{n}\right)\right\}_{n=0}^{\infty}$ converges to $(\bar{x}, \bar{y})$.

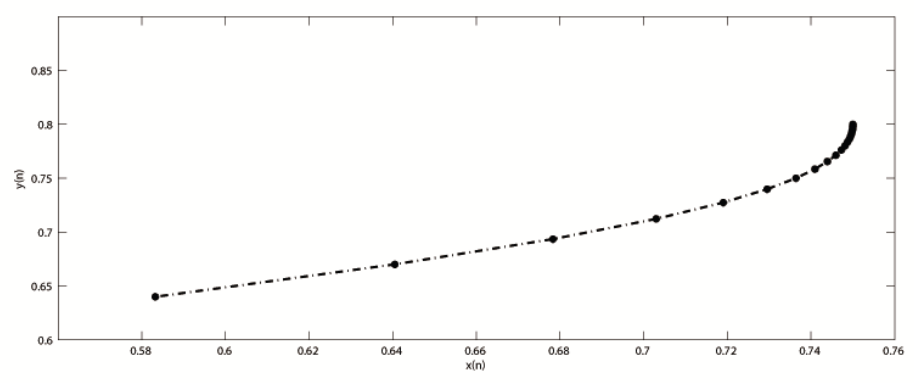

Figure 2. The stability of equilibrium of (1.4) while $\alpha_{i} \leq \beta_{i}$

Example 2. Set $\alpha_{1}=1.5, \alpha_{2}=0.8, \beta_{1}=2, \beta_{2}=1$, Figure 2 shows the stability of equilibrium of (1.4).

\section{References}

[1] H.N. Agiza, A.S. Hegazi and A.A. Elsadany. The dynamics of Bowley's model with bounded rationality. Chaos, Solitons \& Fractals, 12(9):1705-1717, 2001. http://dx.doi.org/10.1016/S0960-0779(00)00021-7.

[2] G.I. Bischi and A. Naimzada. Global analysis of a dynamic duopoly game with bounded rationality. Advances in dynamic games and applications, 5:361-385, 1999.

[3] E.El-Metwally, E.A. Grove, G. Ladas, R. Levins and M. Radin. On the difference equation $x_{n+1}=\alpha+\beta x_{n-1} \mathrm{e}^{-x_{n}}$. Nonlinear Analysis: Theory, Methods $\mathscr{E}$ Applications, 47(7):4623-4634, 2001. http://dx.doi.org/10.1016/S0362$546 \mathrm{X}(01) 00575-2$.

[4] H.A El-Metwally and A.A. Elsadany. Qualitative study of a bounded rationality duopoly game with exponential demand function. Utilitas Mathematica, 88:337$354,2012$.

[5] M.F. Elettreby and H. El-Metwally. On a system of difference equations of an economic model. Discrete Dynamics in Nature and Society, 2013:1611-1617, 2013. http://dx.doi.org/10.1155/2013/405628.

[6] M. Kopel. Simple and complex adjustment dynamics in Cournot duopoly models. Chaos, Solitons \& Fractals, 12(7):2031-2048, 1996. http://dx.doi.org/10.1016/S0960-0779(96)00070-7.

[7] M.R.S. Kulenović and M. Nurkanović. Global behavior of a twodimensional competitive system of difference equations with stocking. Mathematical and Computer Modelling, 55(7-8):1998-2011, 2012. http://dx.doi.org/10.1016/j.mcm.2011.11.059. 
[8] H. Ma, H. Feng, J. Wang and W. Ding. Boundedness and asymptotic behavior of positive solutions for difference equations of exponential form. Journal of nonlinear sciences and applications, 8:893-899, 2015.

[9] G. Papaschinopoulos, G. Ellina and K.B. Papadopoulos. Asymptotic behavior of the positive solutions of an exponential type system of difference equations. Applied Mathematics and Computation, 245:181-190, 2014. http://dx.doi.org/10.1016/j.amc.2014.07.074.

[10] G. Papaschinopoulos, M.A. Radin and C.J. Schinas. On the system of two difference equations of exponetial form: $x_{n+1}=a+b x_{n-1} \mathrm{e}^{-y_{n}}, y_{n+1}=c+$ $d y_{n-1} \mathrm{e}^{-x_{n}}$. Mathematical and Computer Modelling, 54(11-12):2969-2977, 2011. http://dx.doi.org/10.1016/j.mcm.2011.07.019.

[11] T. Puu. Dynamics of Stackelberg duopoly. In: T. Puu and A. Panchuk (eds.), Advances in nonlinear economic dynamics. Nova Science Publishers, New York, 2010 . 